\title{
A local fingerprint for hydrophobicity and hydrophilicity: From methane to peptides
}

Cite as: J. Chem. Phys. 150, 204103 (2019); https://doi.org/10.1063/1.5088418

Submitted: 10 January 2019 . Accepted: 29 April 2019 . Published Online: 22 May 2019

S. Pérez-Conesa (D), Pablo M. Piaggi (D), and Michele Parrinello (D)
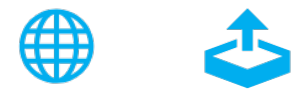

\section{ARTICLES YOU MAY BE INTERESTED IN}

Principal component analysis of nonequilibrium molecular dynamics simulations The Journal of Chemical Physics 150, 204110 (2019); https://doi.org/10.1063/1.5089636

Unsupervised machine learning in atomistic simulations, between predictions and understanding

The Journal of Chemical Physics 150, 150901 (2019); https://doi.org/10.1063/1.5091842

Ions' motion in water

The Journal of Chemical Physics 150, 190901 (2019); https://doi.org/10.1063/1.5090765

\section{Lock-in Amplifiers up to $600 \mathrm{MHz}$}
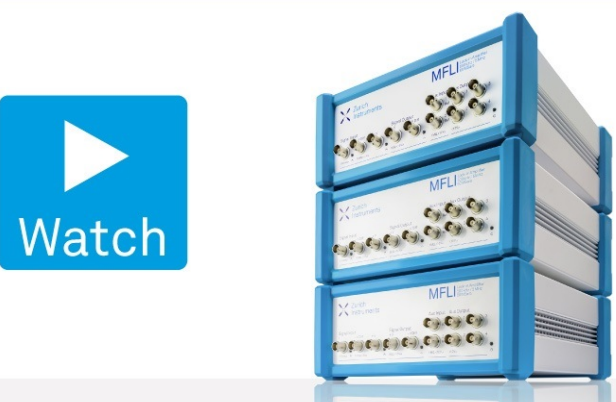

J. Chem. Phys. 150, 204103 (2019); https://doi.org/10.1063/1.5088418 


\title{
A local fingerprint for hydrophobicity and hydrophilicity: From methane to peptides
}

\author{
Cite as: J. Chem. Phys. 150, 204103 (2019); doi: 10.1063/1.5088418 \\ Submitted: 10 January 2019 - Accepted: 29 April 2019 • \\ Published Online: 22 May 2019
}

\section{S. Pérez-Conesa, (D) Pablo M. Piaggi, ${ }^{2,3}$ (D) and Michele Parrinello $0^{3,4, a)}$ (iD}

\author{
AFFILIATIONS \\ ${ }^{1}$ Department of Physical Chemistry, University of Seville, 41012 Seville, Spain \\ ${ }^{2}$ Theory and Simulation of Materials (THEOS), École Polytechnique Fédérale de Lausanne (EPFL), CH-1015 Lausanne, \\ Switzerland \\ ${ }^{3}$ Facoltà di Informatica, Istituto di Scienze Computazionali, and National Center for Computational Design and Discovery of Novel \\ Materials (MARVEL), Università della Svizzera Italiana (USI), Via Giuseppe Buffi 13, CH-6900 Lugano, Switzerland \\ 4Department of Chemistry and Applied Biosciences, ETH Zurich, c/o USI Campus, Via Giuseppe Buffi 13, \\ $\mathrm{CH}-6900$ Lugano, Switzerland
}

a) Electronic mail: parrinello@phys.chem.ethz.ch

\begin{abstract}
An important characteristic that determines the behavior of a solute in water is whether it is hydrophobic or hydrophilic. The traditional classification is based on chemical experience and heuristics. However, this does not reveal how the local environment modulates this important property. We present a local fingerprint for hydrophobicity and hydrophilicity inspired by the two body contribution to the entropy. This fingerprint is an inexpensive, quantitative, and physically meaningful way of studying hydrophilicity and hydrophobicity that only requires as input the water-solute radial distribution functions. We apply our fingerprint to octanol, benzene, and 20 proteinogenic amino acids. Our measure of hydrophilicity is coherent with chemical experience, and moreover, it also shows how the character of an atom can change as its environment is changed. Finally, we use the fingerprint as a collective variable in a funnel metadynamics simulation of a host-guest system. The fingerprint serves as a desolvation collective variable that enhances transitions between the bound and unbound states.
\end{abstract}

Published under license by AIP Publishing. https://doi.org/10.1063/1.5088418

\section{INTRODUCTION}

"Like dissolves like" is one of the earliest chemical rules a scientist learns in relation to solvation. It implies that solutes that are chemically similar to water have a favorable interaction with water and are hydrophilic. On the other hand, solutes that are not like water will tend to repel water and be hydrophobic. ${ }^{1,2}$ Typically, one assigns to each atom its own hydrophobicity or hydrophilicity based on chemical experience and heuristics. Despite the importance of these intuitive classifications, none of them is quantitative nor takes into account thermodynamics or solvent structure. Processes like protein folding, the assembly of molecules, or crystallization depend crucially on their interaction with water. Thus, it would be of great help to have a measure of the hydrophobicity and hydrophilicity of the atoms in a solute molecule and understand how these parameters change as the environment changes. There is a vast and at times controversial literature on the concept of hydrophilicity, and we do not want to enter into this arena, nor do we want to replace what is already available in the literature.

For instance, in the context of protein science, many hydrophobicity scales for amino acids have been proposed based on empirical or computational data without any definitive consensus. ${ }^{3}$ Scales that focus on the hydrophobicity of selected heavy atoms have also been proposed. Some of them are based on local compressibility or density fluctuations of the hydration layers of proteins and surfaces.

Here, we propose to use a concept related to the density fluctuations, namely, the radial distribution function (RDF). Thus, we define a local fingerprint that is a function of the RDF between solute atoms and water oxygen atoms. This fingerprint has been inspired by our previous work on using approximated expressions for the entropy in order to distinguish between solidlike and liquidlike 
environments. ${ }^{6,7}$ We emphasize that the goal of this work is not to calculate the entropy and that we ignore angular correlations that play an important role in a complex liquid such as water. ${ }^{8-20}$ With respect to other hydrophobicity measures, our fingerprint has the advantage of being easy to compute and defined for each atom, and in principle, it can also be experimentally measured. One can thus assess the hydrophobicity of each individual atom and the modifications that result from changes in its environment. We apply this fingerprint to water and methane as representatives of optimal hydrophilicity and hydrophobicity and to more complex systems such as octanol, benzene, and 20 proteinogenic amino acids.

The local fingerprint does not only provide an inexpensive and quantitative assessment of hydrophobicity, but it is also a suitable collective variable $(\mathrm{CV})$ to describe solvation in enhanced sampling simulations. In many cases, solvation and desolvation represent a kinetic bottleneck in spite of not being the main processes under study. This is the case in protein folding, ligand binding, and crystallization. We illustrate the usefulness of the fingerprint in enhanced sampling simulations by using it in a funnel metadynamics simulation of a host-guest system. The fingerprint enhances the transition between the bound and unbound states through a dynamical description of solvation.

\section{FINGERPRINT FOR HYDROPHOBICITY AND HYDROPHILICITY}

Theory provides an expansion of the entropy of a liquid as a sum of many-body correlation functions. ${ }^{21-23}$ Inspired by this theoretical framework, we propose the following term of the expansion as a local fingerprint for hydrophobicity and hydrophilicity of atom $i$ :

$$
S_{\mathrm{s}}^{i}=-2 \pi \rho_{\mathrm{w}, \mathrm{loc}} \int_{0}^{\infty}\left\{g_{i \mathrm{w}}(r) \ln \left[g_{i \mathrm{w}}(r)\right]-g_{i \mathrm{w}}(r)+1\right\} r^{2} d r,
$$

where $\rho_{\mathrm{w}, \text { loc }}$ is the local number density of water and $g_{i \mathrm{w}}(r)$ is the radial distribution function of atom $i$ of the solute and water oxygen atoms. The reader should bear in mind that this is not an expression for the excess entropy of the system but rather one of its contributions. Calculating the entropy requires including higher order terms and angular correlations ${ }^{8-12}$ at a much higher computational cost. This defeats our purpose of having an inexpensive, semiquantitative fingerprint useful also in enhanced sampling simulations. Furthermore, Eq. (1) can be seen in a different light if it is interpreted as a Bregman divergence between $g_{i \mathrm{w}}(r)$ and the perfect gas RDF, i.e., $g(r)=1 \forall r r^{24}$ From this point of view, it represents a distance between these two functions. Equation (1) is also connected to the Kirkwood-Buff ${ }^{5}$ integrals since both are integrals involving the radial distribution function.

It is instructive to calculate the fingerprint value in the simple case of a spherical cavity of radius $R$ embedded in an ideal solvent. In this particular case, $g_{i w}(r)$ in Eq. (1) is

$$
g_{i \mathrm{w}}(r)= \begin{cases}0, & \text { if } r \leq R, \\ 1, & \text { if } r>R .\end{cases}
$$

If we introduce this step function in Eq. (1), the following formula for the fingerprint of a cavity of volume $V=\frac{4}{3} \pi R^{3}$ is obtained:

$$
S_{i}^{\mathrm{cav}}=-\frac{\rho k_{B} V}{2} .
$$

This expression is the leading term of the solvation entropy in the information theory model of hydrophobic interactions ${ }^{1,26,27}$ if one assumes that the solvent behaves ideally.

\section{COMPUTATIONAL METHODS}

All the systems used in this work for unbiased simulations were solutions of a single solute molecule with $1000 \mathrm{SPC} / \mathrm{E}^{28}$ water molecules at water density $0.997 \mathrm{~g} \mathrm{~cm}^{-3}$. The solutes studied were an SPC/E water molecule, methane, n-octanol, benzene, and 20 proteinogenic amino acids. The amino acids were simulated in their standard physiological protonation state and with $\mathrm{N}$-methylated and C-acetylated termini. The OPLS ${ }^{29}$ force field was used for methane and octanol. AMBER03 ${ }^{30}$ was used for the amino acids and benzene. The partial charges of benzene were calculated at the B3LYP/cc-PVTZ level using the ESP method, ${ }^{31}$ and the polarizable continuum model $^{32}$ was used to mimic the aqueous environment. Water molecules were kept rigid using the SETTLE algorithm. ${ }^{33}$ For the rest of the solutes, the bonds involving hydrogen were constrained with the P-LINKS algorithm. ${ }^{34}$ Lennard-Jones cross-term parameters were assigned using $\epsilon_{i j}=\left(\epsilon_{i i} \epsilon_{j j}\right)^{(1 / 2)}$ and $\sigma_{i j}=\frac{\sigma_{i i}+\sigma_{j j}}{2}$, except in the case of AMBER03 where $\sigma_{i j}=\left(\sigma_{i i} \sigma_{j j}\right)^{(1 / 2)}$ was used.

The host-guest system studied by metadynamics simulation was obtained from the SAMPL $5^{35}$ blind contest. The host-guest system studied has code name OAMe/OA-G2, and the structure and topology files used were those provided for the contest. The force fields used were $\mathrm{GAFF}^{36}$ and SPC/E.

All molecular dynamics (MD) simulations were run with GROMACSv5.1.1 ${ }^{37}$ in the NVT ensemble using the stochastic velocity rescaling thermostat ${ }^{38}$ at $298 \mathrm{~K}$ and a relaxation time $\tau=0.1 \mathrm{ps}$. The equations of motion were integrated using the leapfrog algorithm with a 2 fs time step for a total time of $10 \mathrm{~ns}$. The simulation time for the metadynamics simulation was $300 \mathrm{~ns}$ although a shorter simulation would have sufficed to achieve convergence. Periodic boundary conditions were used, and long-range electrostatic interactions were calculated with the PME method. ${ }^{39,40}$ Short range van der Waals interactions were truncated at $10 \AA$.

The calculations of the fingerprint were done using a development version of PLUMED 2. ${ }^{41}$ The RDF is calculated using a kernel density estimation of the radial distribution function, ${ }^{6,7}$ which for a Gaussian kernel is

$$
g_{i \mathrm{w}}(r)=\frac{1}{4 \pi \rho_{\mathrm{w}, \mathrm{loc}} r^{2}} \sum_{j \in \mathrm{w}} \frac{1}{\sqrt{2 \pi \sigma^{2}}} e^{-\left(r-r_{j}\right)^{2} /\left(2 \sigma^{2}\right)},
$$

where $r_{j}$ is the distance between the fingerprinted atom, $i$, and the $j$ th water molecule where $j$ runs over the set of water molecules. $\sigma$ is the Gaussian kernel bandwidth. Kernel density estimation ensures that $g_{i \mathrm{w}}(r)$ is continuous and differentiable with respect to atomic positions for its use as a collective variable in enhanced sampling simulations. In addition, this decreases the noise when the statistics are poor. Nevertheless, a conventional RDF would give identical results. The value of $\sigma$ was $0.05 \AA$ producing RDFs that are smooth but yet preserve all the relevant features. The fingerprint was integrated 
using the trapezoid rule. The upper integration limit was chosen to be $r_{\text {max }}=10 \AA$. Equation (4) corresponds to the single configuration $g_{i \mathrm{w}}(r)$. To reduce noise, $g_{i \mathrm{w}}(r)$ is averaged for its use in Eq. (1).

The local number density of water $\rho_{\mathrm{w}, \text { loc }}$ is generally different from the bulk water density $\rho_{\mathrm{w}}$. This is a consequence of the excluded volume of the solute. For big solutes such as the amino acids considered below, the deviation of $\rho_{\mathrm{w}, \text { loc }}$ from $\rho_{\mathrm{w}}$ can be very significant. For this reason, we have used the local density both in Eqs. (1) and (4). This choice ensures that the RDFs are all equivalently normalized regardless of the excluded volume of the solute.

Well tempered metadynamics (WTMetaD) simulations ${ }^{42,43}$ were run on the host-guest system in its funnel variant. ${ }^{44}$ Funnel metadynamics adds a constant bias potential on the guest such that it remains in a funnel-shaped region with the conical part placed in the cavity of the host and the thin cylindrical region outside host. In this way, the guest diffuses in a region of space where it can easily access the host and not diffuse through all space. The funnel has a length of $23 \AA$ with the cone apex at $15 \AA$ and a cone angle of $45^{\circ}$. The funnel restrain was quadratic with a force constant of $40 \mathrm{~kJ} \AA^{-2}$. The entropy loss due to this restrain is corrected analytically ${ }^{44,45}$ a posteriori using Eq. (1) of the supplementary material.

WTMetaD was performed using two CVs: the inverse of the square root of host-guest contact map and a CV based on the fingerprint $S_{\mathrm{s}}$ that we shall refer to as $S_{\mathrm{CV}}$. Using the inverse of the square root of the contact map ensures that both states are sampled in a balanced fashion. This compensates for the fact that a bound and unbound state have ranges of contact-map values that are very uneven. The chosen contacts are specified in PLUMED's input shown in the supplementary material. $S_{\mathrm{CV}}$ is defined as the sum of $S_{\mathrm{s}}$ of several atoms of both the host and the guest. Only some solute atoms are included for the calculation of $S_{\mathrm{CV}}$ in order to reduce their computational cost. The atoms used can found in Fig. S6 of the supplementary material. An additional simulation without biasing $S_{\mathrm{CV}}$ was performed as a reference.

The WTMetaD simulation was carried out using the same molecular dynamics parameters as the unbiased simulations. The Gaussians were deposited every 1 ps with an initial height of $5 \mathrm{~kJ} \mathrm{~mol}^{-1}$. The Gaussian $\sigma$ s were 0.005 and $0.05 k_{B}$ for the contact map CV and the fingerprint CV. A bias factor of 24 was used. The free energy surfaces were reweighted by the method of Tiwary and Parrinello. ${ }^{46}$ The statistical uncertainties are presented as the standard error of the mean calculated using block averages. Further details of the simulation can be found in the supplementary material.

\section{RESULTS AND DISCUSSION}

\section{A. Simple solutes}

Water and methane are paradigmatic cases of hydrophilic and hydrophobic solutes. Thus, their fingerprint values can be used as references. Water has an $S_{\mathrm{s}}$ of $-1.57 \pm 0.01$ and methane of -2.78 \pm 0.01 . Figure 1 clarifies the physics behind these numbers. The top graph shows the radial distribution functions of the solutes and the bottom graph the integrand $I_{i}(r)$ of the fingerprint

$$
I_{i}(r)=-2 \pi \rho_{\mathrm{W}}\left\{g_{i \mathrm{~W}}(r) \ln \left[g_{i \mathrm{~W}}(r)\right]-g_{i \mathrm{~W}}(r)+1\right\} r^{2} .
$$

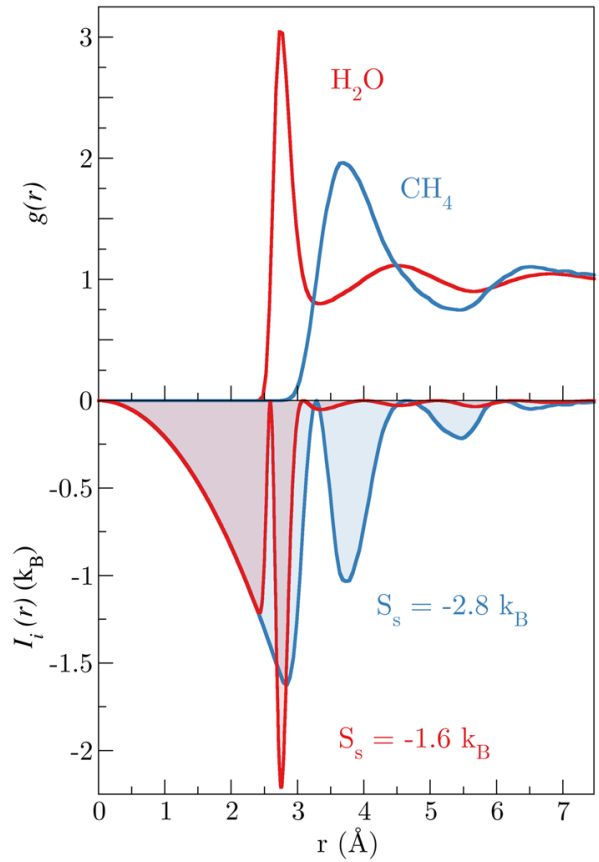

FIG. 1. Top: C-O radial distribution function for the aqueous methane simulation (blue) and 0-0 radial distribution function for a pure water simulation (red). Bottom: for the same pairs, the integrand, $l_{i}(r)$, of the fingerprint is plotted.

The figure shows how $S_{\mathrm{s}}$ varies with the radial structure of the solvent around the solute. In essence, $S_{\mathrm{s}}$ becomes more negative the larger the deviation of the RDF from one. The more the solvent is structured around the solute, the smaller $S_{s}$. Because of the $r^{2}$ factor, the structuring at larger distances is especially effective in decreasing $S_{s}$. At short distances, for $r$ less than a distance $r_{c}$ of the order of the molecular radius, $g_{i \mathrm{w}}(r) \approx 0$ and this small $r$ region gives a contribution proportional to $r_{c}^{3}$. This contribution to $S_{s}$ corresponds to the cavity formation entropy. Methane has a lower $S_{\mathrm{s}}$ than water for two reasons. First, it generates a larger cavity. Second, although its first hydration shell peak is less structured, it is wider; it is located at distances larger than the first hydration shell of water and contains 4 times more water molecules.

We shall use the $S_{\mathrm{s}}$ values for water and methane as representative of extreme hydrophilicity and hydrophobicity. It is therefore convenient to rescale the values of $S_{\mathrm{s}}$ introducing an index $h$ that is +1 for water and -1 for methane. Thus, in this scale, the sign of $h$ determines whether the atom is hydrophobic or hydrophilic.

We now turn to discuss the properties of octanol chosen for its amphiphilic character. Figure 2 shows octanol, water, and methane with their heavy atoms colored according to their $h$ values. The index clearly distinguishes between hydrophobic and hydrophilic atoms. The trend in $h$ values is in accordance with what could have been expected. The index can also deal with intermediate cases as the carbon atom attached to the alcohol group. This atom should be labeled as less hydrophobic than aliphatic carbons due to its partial positive 


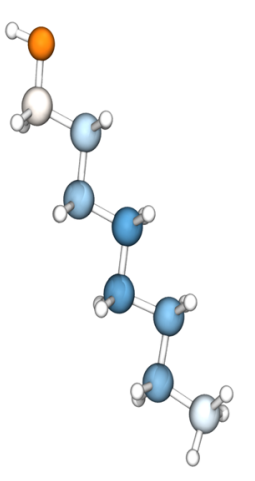

Octanol

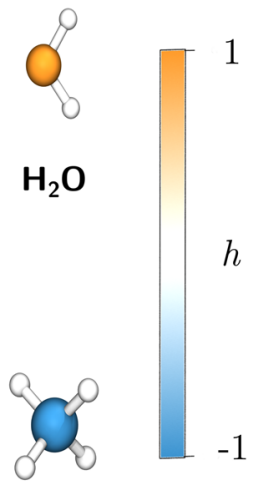

$\mathrm{CH}_{4}$
FIG. 2. Octanol, water, and methane molecules with their heavy atoms colored according to the $h$ index. The scale ranges from hydrophobic (blue), to intermediate (white), and to hydrophilic (orange)

charge generated by the electronegativity difference with the oxygen it is bonded to. Nevertheless, since the influence of a hydrophilic atom on the fingerprint of others is limited, the fingerprint is local with respect to the atoms of the molecule. The terminal $\mathrm{CH}_{3}$ has a lower $h$ than the $\mathrm{CH}_{2}$ carbons. This can be ascribed to the fact that the solvation shells of neighboring $\mathrm{CH}_{2}$ groups in the aliphatic chain overlap. This shifts the RDF first solvation peak to higher distances thus increasing $h$. Since $\mathrm{CH}_{3}$ has only one neighbor, this effect is less pronounced. Figure S3 of the supplementary material illustrates this by analyzing the RDFs of primary, secondary, and tertiary carbon atoms and methane. Figure S4 of the supplementary material includes the numeric values of the fingerprint of the atoms in octanol.

An interesting case is that of ions, in which their classification into hydrophilic or hydrophobic could be misleading. The fingerprint $S_{\mathrm{s}}$ for $\mathrm{Na}^{+}$is $-3.9 \mathrm{k}_{\mathrm{B}}$ which would mistakenly classify it as more hydrophobic than methane. This is mostly due to the intensity of the first shell peak of the $\mathrm{Na}^{+}-\mathrm{H}_{2} \mathrm{O}$ RDF which decreases strongly the value of $S_{\mathrm{s}}$ because of the strong interaction with the ion (Fig. S2 of the supplementary material). In the classical electrochemistry or coordination chemistry notion of the hydrated ion, ${ }^{47}$ we consider the ion and its first hydration shell as the solute. In this context, we can consider the sodium cation as a buried atom and the first hydration shell atoms as the solvent exposed atoms in which to measure the fingerprint. This concept has been useful in the development of metal ion force fields. ${ }^{48,49}$ The first-shell water molecules have an $S_{\mathrm{s}}$ of $-0.9 \mathrm{k}_{\mathrm{B}}$ which is more hydrophilic than bulk water. Therefore, if we use the hydrated ion as the solute, we can conclude the $\mathrm{Na}^{+}$hydrated ion is hydrophilic as expected.

\section{B. Amino acids}

The fingerprint for the heavy atoms of the 20 proteinogenic amino acids was computed, offering the possibility of testing our fingerprint on a wide range of chemical groups. This is a first step for future use in the study of hydrophobic and hydrophilic interactions in proteins. Figure 3 shows the different amino acid molecules with the heavy atoms in the side chains colored according to their $h$ value. The backbone atoms are shown only for glycine, but a similar picture is obtained for the other amino acids. As in the case of octanol, $h$ assigns a hydrophobic value to aliphatic carbons and a hydrophilic value to polar $\mathrm{N}$ and $\mathrm{O}$ atoms of hydrophilic residues. All the heavy atoms of the backbone have $h$ values adequate to the hydrophilicity or hydrophobicity that chemical intuition suggests. A list of $h$ values can be found in Fig. S5 of the supplementary material.

While most of the $h$ values reflect the expected behavior, some apparently surprising values can be seen. For instance, the aromatic $\mathrm{C}$ are placed in the middle of the $h$ scale, and thus, they are classified as neither properly hydrophilic nor hydrophobic. In reality, this result is in line with the known solvation behavior of benzene which is much more soluble than its aliphatic counterpart cyclohexane. The reasons for this effect have been discussed in the literature. ${ }^{50-53}$ As seen from the point of view of our fingerprint, this results from the fact that the other atoms in the ring exclude some of the solvation water leading to a reduction in the RDF peak height. In order to confirm that this behavior is not an artifact of our force field, we have calculated $S_{\mathrm{s}}$ using the benzene RDF kindly provided to us by Choudary and Chandra obtained using ab initio MD. ${ }^{54}$ The ab initio value, $S_{\mathrm{s}}=-1.9 \mathrm{k}_{\mathrm{B}}$, is very close to that of the AMBER force field. Here, we did not scale the $S_{\mathrm{s}}$ values since we do not have the ab initio reference point for methane.

Another $h$ value that deserves some discussion is that of the sulfur atoms with $h \sim 0$. This can be linked to the fact that the electronegativity of sulfur is intermediate between carbon and oxygen and to the ability of sulfur to accept weak $\mathrm{H}$ bonds. ${ }^{55,56}$

Since we relate $h$ to the water solvation structure and the water structure around each atom and the conformation of the solute can fluctuate as a function of time, we also looked at the distribution of this index. We consider the $h$ value obtained from RDF averaged over a 400 ps moving window to allow the fingerprint to vary and study its distribution. The data obtained from all the amino acid simulations were put in a histogram in which we considered separately aliphatic $\mathrm{C}$, aromatic $\mathrm{C}, \mathrm{S}$, and $\mathrm{O}$, and $\mathrm{N}$ of the side chains. The histograms are shown in Fig. 4.

In the histogram, the hydrophobic aliphatic $\mathrm{C}$ are clearly separated from the hydrophilic $\mathrm{O}$ and $\mathrm{N}$ of the side chains, proving the usefulness of the fingerprint. As discussed previously, the distribution of the aromatic $\mathrm{C}$ and $\mathrm{S}$ are centered around $h \sim 0$. The distribution of the hydrophilic $\mathrm{O}$ and $\mathrm{N}$ of the side chains (shown in red in Fig. 4) presents two peaks and a shoulder. The peak at $h \sim 0.8$ corresponds to all the hydrophilic $\mathrm{O}$ and $\mathrm{N}$ of charged amino acids with the exception of arginine, while the other peak at $h \sim 1.4$ corresponds to hydrophilic $\mathrm{O}$ and $\mathrm{N}$ of neutral amino acids and arginine. Charged residues have a lower $h$ than neutral ones because they induce more structure in water. Arginine is an exception to this rule due to its higher charge delocalization and therefore leads to a less well-defined solvation structure. The shoulder at $0<h<0.6$ in the histogram of $\mathrm{O}$ and $\mathrm{N}$ of the side chains corresponds to glutamate since carboxylate oxygen atoms have a very negative effective charge with respect to the rest of hydrophilic atoms. The histogram of hydrophobic aliphatic C has two peaks. The peak at $h \sim-0.4$ 
Proteinogenic Amino Acids
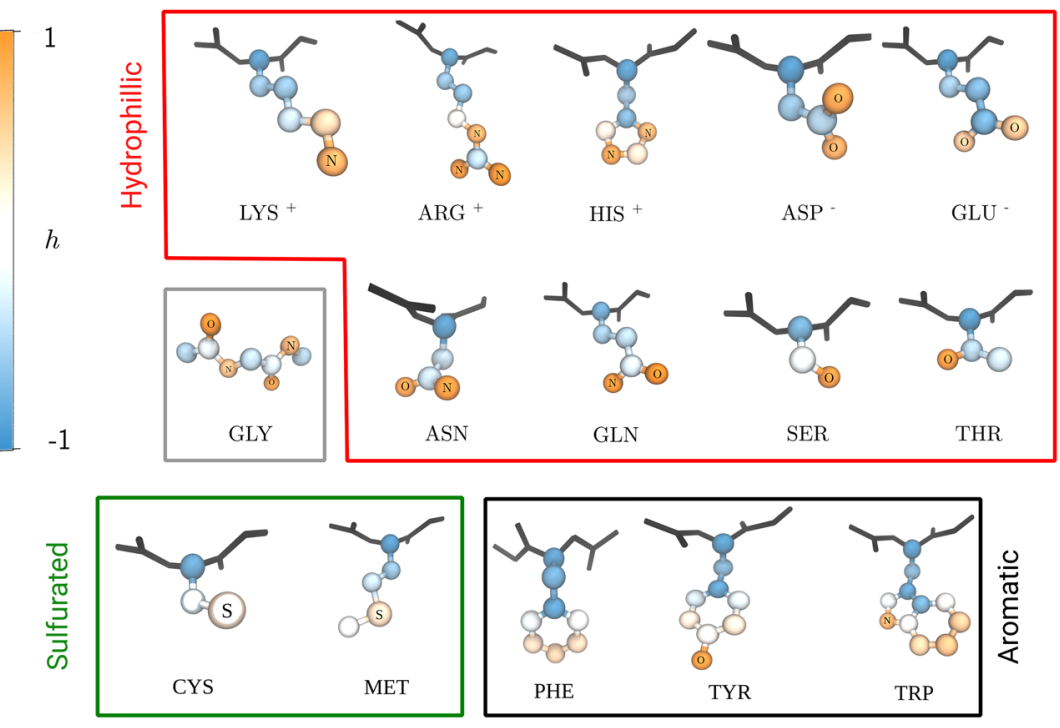

FIG. 3. Structures of the proteinogenic amino acids with their heavy atoms colored according to the $h$ index. The scale ranges from hydrophobic (blue) to intermediate (white) and to hydrophilic (orange). Unlabeled atoms are carbon. Hydrogen atoms are omitted. Since all backbone atoms have similar $h$ index, only side chain atoms are considered. Backbone atoms are visible for glycine (gray box). The boxes organize the amino acids by families: hydrophilic (red), glycine (gray), sulfur-containing (green), aromatic (black), and hydrophobic (blue).
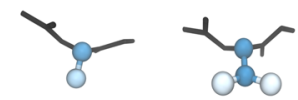

ALA

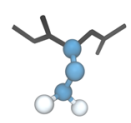

LEU

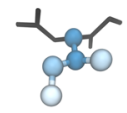

ILE

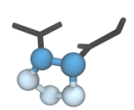

PRO

\section{$\frac{0}{0}$
음
$\frac{1}{0}$
는
조}

corresponds to $\mathrm{CH}_{3}$ carbon atoms, while the broad peak at $h \sim-1.75$ corresponds to $\mathrm{CH}_{2}$ and $\mathrm{CH}$ carbon atoms. This behavior has been discussed earlier in Sec. IV A for octanol. The histogram for aromatic $\mathrm{C}$ shows three peaks. The two peaks around $h \sim 0.25$ correspond to the more solvent exposed aromatic $\mathrm{C}$, while the remaining peak centered at $h \sim-1.25$ corresponds to $\mathrm{C}$ closer to the $\mathrm{C} \beta$.
It is also interesting to test whether our fingerprint correlates with the notion of hydrophilicity given by the free energy of solvation. In Fig. S1 of the supplementary material, we study the correlation between the maximum $h$ value of the amino acids side chain with the experimental free energy of solvation. ${ }^{57}$ The correlation is satisfactory with correlation coefficient $\left(R^{2}\right) 0.57$.

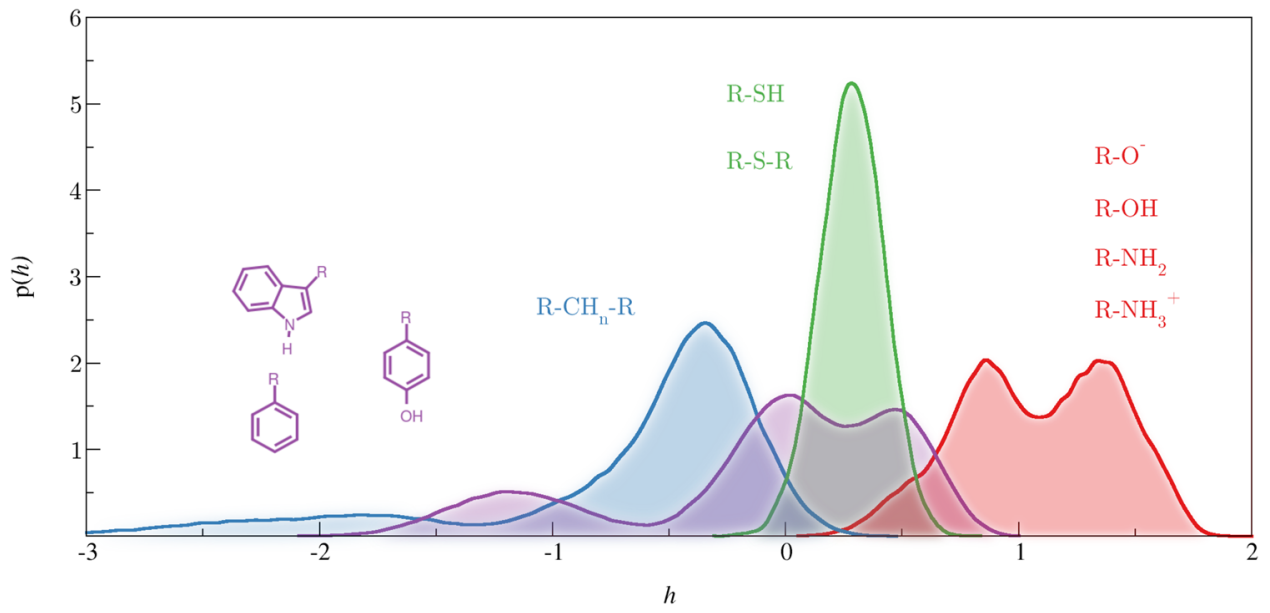

FIG. 4. Probability densities of the hydrophobicity fingerprint, $h$, of different groups of atoms in their respective simulations. The lines are the distributions of $h$ for $C$ atoms of hydrophobic amino acids (blue), $\mathrm{N}$ and $\mathrm{O}$ atoms of hydrophilic amino acids (red), aromatic C atoms (purple), and $\mathrm{S}$ atoms (green). 


\section{Enhanced sampling simulations}

In Subsections IV $\mathrm{A}$ and IV $\mathrm{B}$, we used the fingerprint to describe the hydrophobicity and hydrophilicity of different solutes. In this section, we will show that the fingerprint can also be used as a collective variable to describe solvation in enhanced sampling simulations. Figure 5(b) shows the system chosen for the funnel WTMetaD simulations. It is a host-guest system consisting of a barrel-shaped host molecule and a ligand guest molecule that can fit in the cavity.

As in many ligand-protein systems, desolvation is a key collective variable and a kinetic barrier to the binding if unbiased. If only the contact map is biased, the guest has to wait close to the entrance of the host until it desolvates and binding can happen (Fig. S8 of the supplementary material). As a consequence, the simulation lacks diffusion in CV space and the simulation's convergence is compromised. This has been observed for this system in previous metadynamics simulations by Bhakat and Söderhjelm. ${ }^{58}$ Their solution was to add a static bias potential that desolvates the interior of the host during the metadynamics and then correct the free energy of binding with a desolvation free energy term obtained from a separate free energy perturbation simulation.

Here, we bias two CVs with WTMetaD: the inverse of the square root of host-guest contact map and a CV based on the fingerprint $S_{\mathrm{s}}$ that we shall refer to as $S_{\mathrm{CV}}$. This results in convergence of the simulation and free diffusion of the system from bound to unbound (Fig. S8). $S_{\mathrm{CV}}$ acts as desolvation CV that allows the host and guest to desolvate during the binding process. From the simulation, we calculate the free energy surface (FES) as a function of the vertical distance between the centers of the guest and the bottom atoms of the host, $z$, and $S_{\mathrm{CV}}$. The FES is plotted in Fig. 5(a). In state (1), the guest is unbound and fully solvated. Along the diagonal path from (2) to (3), the guest is about $6 \AA$ away from its bound position. The guest and host desolvate at the same time the guest enters the host. We can interpret this as the guest forcing water molecules out of the host-guest adduct as it is drawn by intermolecular forces into the opening of the barrel. Finally, from (3) to (4), there is a desolvation of the host and guest at nearly constant $z$. This is a situation in which the guest is at the host's doormat but requires a fluctuation of the solvent in order for there to be room in the host to enter. Our interpretation of the $S_{\mathrm{CV}}$ as a desolvation $\mathrm{CV}$ is supported by the mirroring of the presented FES and an equivalent FES using the number of water molecules in the barrel instead of $S_{\mathrm{CV}}$. This FES is shown in Fig. S7 of the supplementary material.

Finally, the free energy of binding of the host to the guest is $-28.1 \pm 0.8 \mathrm{~kJ} \mathrm{~mol}^{-1}$. This results from a projection of the FES onto $z$ and the entropy correction of the funnel. This result is close to the experimental value $-21.6 \mathrm{~kJ} \mathrm{~mol}^{-159}$ and statistically identical to the value obtained by Bhakat and Söderhjelm ${ }^{58}$ using a different simulation protocol.

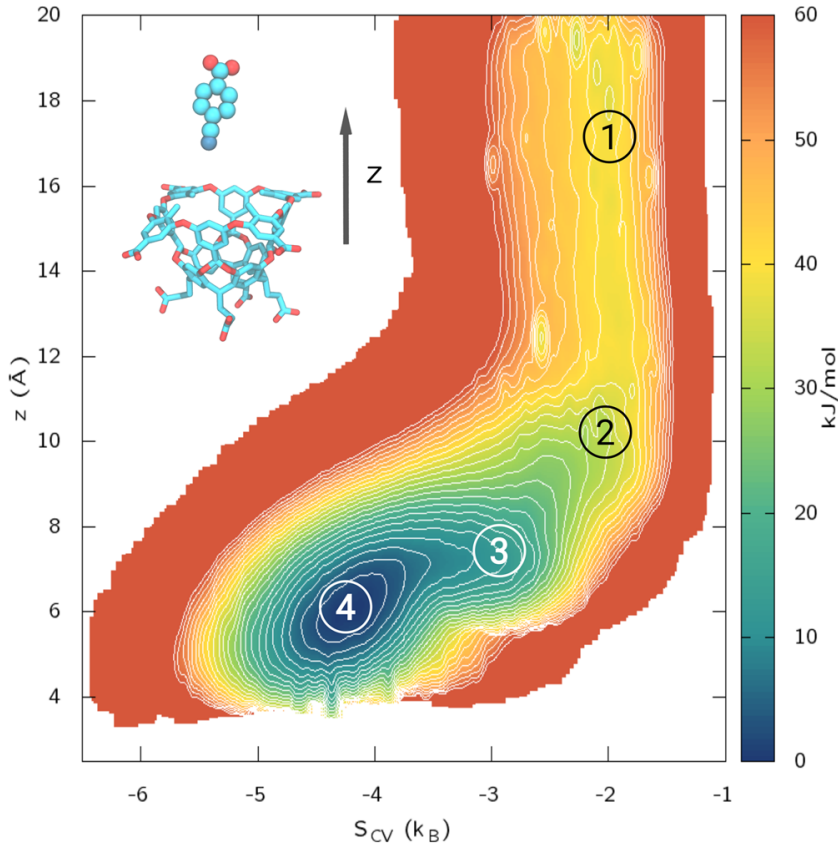

a)

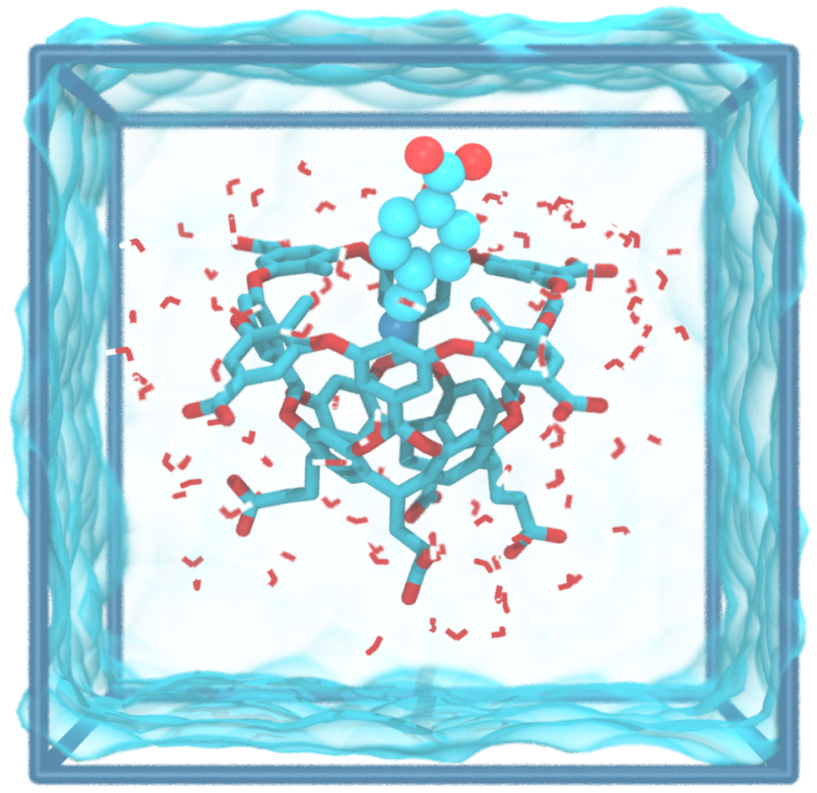

b)

FIG. 5. (a) Reweighted free energy surface of the host-guest system as a function of the vertical distance between the centers of the guest and the bottom atoms of the host, $z$, and the fingerprint collective variable. (b) Schematic (not to scale) representation of the host-guest system (OAMe-OAG2 in the SAMPL $5^{35}$ contest). The solvation is represented by the surface and some of the water molecules are explicitly depicted. 


\section{CONCLUSIONS}

We have developed a local fingerprint for hydrophobicity and hydrophilicity. The fingerprint is inspired by the two body solute water contributions to the entropy, which is a function of the RDF. In this context, whether an atom is hydrophobic or hydrophilic is a consequence of the structure of water around it. This feature allows us to understand how the character of a solute is modulated by its environment. We have also introduced an index of hydrophilicity $h$ that uses methane and water as representatives of hydrophobic and hydrophilic behavior. We show the usefulness of the fingerprint in enhanced sampling simulations by studying a host-guest system in which the fingerprint serves as a desolvation CV and allows for fast transition between the bound and unbound states. We expect that the fingerprint could also provide insight into more complex phenomena where hydrophobicity plays an important role, such as protein folding.

\section{SUPPLEMENTARY MATERIAL}

See supplementary material for a PDF file containing additional information including extra radial distribution functions, fingerprint integrands, numeric values of the local fingerprint, free energy surfaces, and metadynamics details not displayed in the text.

\section{ACKNOWLEDGMENTS}

The authors thank Ashu Choudhary and Amalendu Chandra for kindly providing us with their $a b$ initio benzene RDF. The authors also thank Enrique Sánchez Marcos, Riccardo Capelli, and Tarak Karmakar for helpful discussions. S.P.-C. also acknowledges the Spanish Ministry of Education, Culture and Sports for the Ph.D. Grant (No. FPU14/02100). P.M.P. and M.P. acknowledge support from the NCCR MARVEL funded by the Swiss National Science Foundation and from European Union Grant No. ERC-2014-AdG670227/VARMET. The computational time for this work was provided by the Swiss National Supercomputing Center (CSCS) under Project ID p503.

\section{REFERENCES}

${ }^{1}$ D. Chandler, Nature 437, 640 (2005).

${ }^{2}$ K. Lum, D. Chandler, and J. D. Weeks, J. Phys. Chem. B 103, 4570 (1999).

${ }^{3}$ S. Simm, J. Einloft, O. Mirus, and E. Schleiff, Biol. Res. 49, 31 (2016).

${ }^{4}$ P. J. Rossky, Faraday Discuss. 146, 13 (2010); e-print arXiv:1009.4658.

${ }^{5}$ E. Xi, V. Venkateshwaran, L. Li, N. Rego, A. J. Patel, and S. Garde, Proc. Natl, Acad. Sci. U. S. A. 114, 13345 (2017).

${ }^{6}$ P. M. Piaggi, O. Valsson, and M. Parrinello, Phys. Rev. Lett. 119, 015701 (2017).

${ }^{7}$ P. M. Piaggi and M. Parrinello, J. Chem. Phys. 147, 114112 (2017).

${ }^{8}$ T. Lazaridis and M. E. Paulaitis, J. Phys. Chem. 96, 3847 (1992).

${ }^{9}$ T. Lazaridis and M. E. Paulaitis, J. Phys. Chem. 98, 635 (1994).

${ }^{10}$ T. Lazaridis, J. Phys. Chem. B 104, 4964 (2000).

${ }^{11}$ M. Kinoshita, N. Matubayasi, Y. Harano, and M. Nakahara, J. Chem. Phys. 124, 024512 (2006).

${ }^{12}$ M. Liu, Q. A. Besford, T. Mulvaney, and A. Gray-Weale, J. Chem. Phys. 142, 114117 (2015).

${ }^{13}$ R. M. Lynden-Bell and J. C. Rasaiah, J. Chem. Phys. 107, 1981 (1997).

${ }^{14}$ D. L. Bergman, A. P. Lyubartsev, and A. Laaksonen, Phys. Rev. E 60, 4482 (1999).

${ }^{15}$ T. Lazaridis, J. Phys. Chem. B 102, 3531 (1998).
${ }^{16}$ C. N. Nguyen, T. Kurtzman Young, and M. K. Gilson, J. Chem. Phys. 137, 044101 (2012).

${ }^{17} \mathrm{Z} . \mathrm{Li}$ and T. Lazaridis, Computer Drug Discovery and Design (Springer, 2012), pp. 393-404.

${ }^{18}$ R. Abel, T. Young, R. Farid, B. J. Berne, and R. A. Friesner, J. Am. Chem. Soc. 130, 2817 (2008).

${ }^{19}$ D. J. Huggins and M. C. Payne, J. Phys. Chem. B 117, 8232 (2013).

${ }^{20}$ C. N. Nguyen, A. Cruz, M. K. Gilson, and T. Kurtzman, J. Chem. Theory Comput. 10, 2769 (2014).

${ }^{21}$ H. S. Green, The Molecular Theory of Fluids (North-Holland Publishing Company, Amsterdam, 1952).

${ }^{22}$ R. E. Nettleton and M. S. Green, J. Chem. Phys. 29, 1365 (1958).

${ }^{23}$ A. Baranyai and D. J. Evans, Phys. Rev. A 40, 3817 (1989).

${ }^{24}$ P. M. Piaggi and M. Parrinello, Proc. Natl. Acad. Sci. U. S. A. 115, 10251 (2018).

${ }^{25}$ J. G. Kirkwood and F. P. Buff, J. Chem. Phys. 19, 774 (1951).

${ }^{26}$ S. Garde, G. Hummer, A. E. García, M. E. Paulaitis, and L. R. Pratt, Phys. Rev. Lett. 77, 4966 (1996).

${ }^{27}$ G. Hummer, S. Garde, A. E. Garcia, A. Pohorille, and L. R. Pratt, Proc. Natl. Acad. Sci. U. S. A. 93, 8951 (1996).

${ }^{28}$ H. J. C. Berendsen, J. R. Grigera, and T. P. Straatsma, J. Phys. Chem. 91, 6269 (1987).

${ }^{29}$ G. A. Kaminski, R. A. Friesner, J. Tirado-Rives, and W. L. Jorgensen, J. Phys. Chem. B 105, 6474 (2001).

${ }^{30}$ Y. Duan, C. Wu, S. S. Chowdhury, M. C. Lee, G. Xiong, W. Zhang, R. Yang, P. Cieplak, R. Luo, T. Lee, J. Caldwell, J. Wang, and P. Kollman, J. Comput. Chem. 24, 1999 (2003).

${ }^{31}$ B. H. Besler, K. M. Merz, and P. A. Kollman, J. Comput. Chem. 11, 431 (1990).

${ }^{32}$ J. Tomasi, B. Mennucci, and R. Cammi, Chem. Rev. 105, 2999 (2005).

${ }^{33}$ S. Miyamoto and P. A. Kollman, J. Comput. Chem. 13, 952 (1992).

${ }^{34}$ B. Hess, J. Chem. Theory Comput. 4, 116 (2008).

${ }^{35}$ J. Yin, N. M. Henriksen, D. R. Slochower, M. R. Shirts, M. W. Chiu, D. L. Mobley, and M. K. Gilson, J. Comput.-Aided Mol. Des. 31, 1 (2017).

${ }^{36}$ J. Wang, R. M. Wolf, J. W. Caldwell, P. A. Kollman, and D. A. Case, J. Comput. Chem. 25, 1157 (2004); e-print arXiv:z0024.

${ }^{37}$ M. J. Abraham, T. Murtola, R. Schulz, S. Páll, J. C. Smith, B. Hess, and E. Lindah, SoftwareX 1-2, 19 (2015); e-print arXiv:1503.05249v1.

${ }^{38}$ G. Bussi, D. Donadio, and M. Parrinello, J. Chem. Phys. 126, 014101 (2007); e-print arXiv:0803.4060v1.

${ }^{39}$ T. Darden, D. York, and L. Pedersen, J. Chem. Phys. 98, 10089 (1993); e-print arXiv:9807099 [cond-mat].

${ }^{40}$ U. Essmann, L. Perera, M. L. Berkowitz, T. Darden, H. Lee, and L. G. Pedersen, J. Chem. Phys. 103, 8577 (1995).

${ }^{41}$ G. A. Tribello, M. Bonomi, D. Branduardi, C. Camilloni, and G. Bussi, Comput. Phys. Commun. 185, 604 (2014).

${ }^{42}$ A. Barducci, G. Bussi, and M. Parrinello, Phys. Rev. Lett. 100, 020603 (2008).

${ }^{43}$ J. F. Dama, M. Parrinello, and G. A. Voth, Phys. Rev. Lett. 112, 240602 (2014).

${ }^{44}$ V. Limongelli, M. Bonomi, and M. Parrinello, Proc. Natl. Acad. Sci. U. S. A. 110, 6358 (2013); e-print arXiv:1408.1149.

${ }^{45}$ T. W. Allen, O. S. Andersen, and B. Roux, Proc. Natl. Acad. Sci. U. S. A. 101, 117 (2004).

${ }^{46}$ P. Tiwary and M. Parrinello, J. Phys. Chem. B 119, 736 (2015).

${ }^{47}$ D. T. Richens, The Chemistry of Aqua Ions (J. Wiley, West Sussex, England, 1997).

${ }^{48}$ J. M. Martínez, R. R. Pappalardo, and E. Sánchez Marcos, J. Am. Chem. Soc. 121, 3175 (1999).

${ }^{49}$ E. Galbis, J. Hernández-Cobos, C. den Auwer, C. Le Naour, D. Guillaumont, E. Simoni, R. R. Pappalardo, and E. Sánchez Marcos, Angew. Chem. 122, 3899 (2010).

${ }^{50}$ C. McAuliffe, J. Phys. Chem. 70, 1267 (1966).

${ }^{51}$ S. Cabani, P. Gianni, V. Mollica, and L. Lepori, J. Solution Chem. 10, 563 (1981).

${ }^{52}$ D. Ben-Amotz, Annu. Rev. Phys. Chem. 67, 617 (2016).

${ }^{53}$ R. C. Harris and B. M. Pettitt, J. Phys.: Condens. Matter 28, 083003 (2016). 
${ }^{54}$ A. Choudhary and A. Chandra, Phys. Chem. Chem. Phys. 18, 6132 (2016).

${ }^{55}$ H. S. Biswal, P. R. Shirhatti, and S. Wategaonkar, J. Phys. Chem. A 114, 6944 (2010).

${ }^{56}$ V. Rao Mundlapati, S. Ghosh, A. Bhattacherjee, P. Tiwari, and H. S. Biswal, J. Phys. Chem. Lett. 6, 1385 (2015).
${ }^{57}$ R. Wolfenden, L. Andersson, P. Cullis, and C. Southgate, Biochemistry 20, 849 (1981).

${ }^{58}$ S. Bhakat and P. Söderhjelm, J. Comput.-Aided Mol. Des. 31, 119 (2017).

${ }^{59}$ M. R. Sullivan, P. Sokkalingam, T. Nguyen, J. P. Donahue, and B. C. Gibb, J. Comput.-Aided Mol. Des. 31, 21 (2017). 